\title{
Streamwise Velocity Statistics in Turbulent Boundary Layers that Spatially Develop to High Reynolds Number
}

\author{
P. Vincenti - J. Klewicki - C. Morrill-Winter • C.M. White • M. Wosnik
}

Received: date / Accepted: date

\begin{abstract}
Well-resolved measurements of the streamwise velocity in zero pressure gradient turbulent boundary layers are presented for friction Reynolds numbers up to 19,670. Distinct from most studies, the present boundary layers undergo nearly a decade increase in Reynolds number solely owing to streamwise development. The profiles of the mean and variance of the streamwise velocity exhibit logarithmic behavior in accord with other recently reported findings at high Reynold number. The inner and mid-layer peaks of the variance profile are evidenced to increase at different rates
\end{abstract}

P. Vincenti

Department of Mechanical Engineering

University of New Hampshire

Durham, NH, 03824 E-mail: p.vincenti@unh.edu

\section{J. Klewicki}

Department of Mechanical Engineering

University of New Hampshire

Durham, NH, 03824, USA

and

Department of Mechanical Engineering

University of Melbourne

Melbourne, Victoria, 3010, Australia

E-mail: joe.klewicki@unh.edu

C. Morrill-Winter

Department of Mechanical Engineering

University of Melbourne

Melbourne, Victoria, 3010, Australia

E-mail: caleb.morrillwinter@gmail.com

C.M. White

Department of Mechanical Engineering

University of New Hampshire

Durham, NH, 03824

E-mail: Chris.White@unh.edu

M. Wosnik

Department of Mechanical Engineering

University of New Hampshire

Durham, NH, 03824

E-mail: Martin.Wosnik@unh.edu with increasing Reynolds number. A number of statistical features are shown to correlate with the position where the viscous force in the mean momentum equation loses leading order importance, or similarly, where the mean effect of turbulent inertia changes sign from positive to negative. The near-wall peak region in the 2D spectrogram of the fluctuations is captured down to wall-normal positions near the edge of the viscous sublayer at all Reynolds numbers. The spatial extent of this near-wall peak region is approximately invariant under inner normalization, while its large wavelength portion is seen to increase in scale in accord with the position of the mid-layer peak, which resides at a streamwise wavelength that scales with the boundary layer thickness.

Keywords turbulent boundary layer · high Reynolds number $\cdot$ streamwise velocity statistics

\section{Introduction}

The structure of the mean flow and turbulence in the zero pressure gradient turbulent boundary layer has been a subject of both fundamental and applied research for many decades, e.g., see the review articles by Gad-el-Hak \& Bandyopadhyay (1994) and Klewicki (2010). Because of their technological importance, high Reynolds number turbulent boundary layers continue to be studied intensely. While considerable progress has been made during the last decade, a number of issues pertaining to the Reynolds number scaling behaviors of the boundary layer remain unsettled, e.g., Marusic et al. (2010); Klewicki (2010); Smits, McKeon \& Marusic (2011).

Acquiring the data needed to systematically clarify the properties of wall-turbulence as the Reynolds number becomes large poses two primary challenges. 
The first is to attain the desired high Reynolds number conditions. The second is that the experiment must sufficiently resolve (spatially and temporally) the turbulence in the boundary layer. In practice, the requisite attributes of the facility and sensors are connected because there is a relative scale-separation between the largest and smallest dynamical motions with increasing Reynolds number. This is perhaps most easily seen by employing the Reynolds number based upon the boundary layer thickness and the friction velocity,

$\delta^{+}=\frac{\delta}{\nu / u_{\tau}}=\frac{u_{\tau} \delta}{\nu}$

where $\nu$ is the kinematic viscosity, $\delta$ denotes $\delta_{99}$ and $u_{\tau}=\sqrt{\tau_{w} / \rho}$. Here $\tau_{w}$ is the mean wall shear stress, and $\rho$ is the mass density. Equation 1 nominally shows that the $O(\delta)$ (largest) scales of motion become relatively larger than the $O\left(\nu / u_{\tau}\right)$ (smallest) scales at a rate that is directly proportional to the Reynolds number.

Equation 1 also shows that increases in Reynolds number can be attained by increasing $u_{\tau}$ (and thus the flow speed), decreasing $\nu$, or increasing $\delta$ (and thus the flow development length), or some suitable combination. The first two, however, cause a reduction in the physical length scale, $\nu / u_{\tau}$, while the third increases $\delta$ relative to $\nu / u_{\tau}$. These considerations are important in the design of high Reynolds number experiments, since any given sensor (or sensing volume) has a finite dimension.

The spatial and temporal resolution of hotwire sensors, as associated with the study of turbulent boundary layers, has been studied extensively for a number of years, e.g., Johansson \& Alfredsson (1983); Ligrani \& Bradshaw (1987); Klewicki \& Falco (1990); Hutchins et al. (2009). These studies indicate, for example, that spatial averaging effects (caused by finite probe size) have observable influences on the streamwise velocity variance derived from single wire sensors for normalized wire lengths, $\ell^{+}=\ell u_{\tau} / \nu$, as small as about 5 , but that a fixed $\ell^{+}$sensor captures relatively more of the broadband signal as $\delta^{+}$increases. For example, the attenuation formula of Hutchins et al. (2009), which includes both $\ell^{+}$and $\delta^{+}$effects (see their Eqn. 3.1), estimates that at $\delta^{+}=5,000$ an $\ell^{+}=25$ sensor will attenuate the near-wall peak value of $\left\langle u^{2}\right\rangle^{+}$by about $11.3 \%$, and at $\delta^{+}=20,000$ the attenuation of an $\ell^{+}=25$ sensor will be about $9.5 \%$. Note that $\tilde{u}$ is the instantaneous signal, and $U$ and $u$ are the mean and fluctuation, respectively.

Spatial averaging induced signal attenuation has complicated elucidation of the Reynolds number dependence of $\left\langle u^{2}\right\rangle^{+}$. This partly stems from it typically being most convenient to increase $\delta^{+}$by increasing the freestream velocity, $U_{\infty}$, and consequently, increases in $\delta^{+}$are accompanied by an increase in $\ell^{+}$. Thus, studies exploring the $\delta^{+}$dependence of the near-wall peak in $\left\langle u^{2}\right\rangle^{+}$that only consider well-resolved measurements reveal a log linear increase with $\delta^{+}$, e.g., Klewicki \& Falco (1990); Metzger \& Klewicki (2001); Hutchins et al. (2009), while those that do not control for this influence do not, e.g., Mochizuki \& Nieuwstadt (1996).

Insufficient temporal resolution can also lead to phenomena similar to that associated with spatial attenuation (Hutchins et al., 2009). For an anemometer bridge and probe combination of sufficient frequency response, temporal resolution depends on the analog-to-digital sampling rate, and errors here primarily influence the capacity to accurately capture the spectral properties of the underlying signal. Hutchins et al. estimate that, for $u$ measurements, the highest frequency in the flow corresponds to about 3 viscous time scales, and thus the inner-normalized cut-off frequency, $f_{c}^{+}=\nu f_{c} / u_{\tau}^{2}$, should have a value in excess of about $1 / 3$.

The number of studies that report reliable mean velocity profiles for $\delta^{+} \geq 10,000$ is substantial, e.g., see Monkewitz, Chauhan \& Nagib (2008) and the references therein. Conversely, there currently exists only a small number of well-resolved $u$ measurements from controlled facilities at these Reynolds numbers. Notable among these are the single sensor hotwire measurements of Hutchins et al. (2009) and Kulandaivelu (2012) from the High Reynolds Number Boundary Layer Wind Tunnel (HRNBLWT) at the University of Melbourne, and the measurements made by DeGraaff \& Eaton (2000) in the Stanford University pressurized wind tunnel. The Stanford experiments employed a specially designed laser Doppler velocimetry system. Data comparisons herein primarily use the measurements from the HRNBLWT, since these profiles are exceptionally smooth (owing partly to very long averaging times), and cover a Reynolds number range comparable to that of the present study.

For brevity, the specific attributes of the statistical quantities explored are not reviewed here, but rather are primarily introduced and discussed during the data presentation. In these presentations, some features appear to correlate with scaling behaviors that Wei et al. (2005) found for the terms in the mean momentum equation. Thus, for reference we present these behaviors in Table 1. For the flat plate flow, the mean dynamical equation contains terms representing the mean flow inertia (MI), the mean effect of the turbulent inertia (TI) and the mean viscous force (VF). Note that the TI term crosses zero within layer III, and thus, when interpreted as a force, this term behaves like a momentum source in layer II and a momentum sink in layer IV. The outer edge of layer III locates the position from the 
Table 1 Leading order balances and width scalings of the layers associated with the mean momentum equation (Wei et al., 2005).

\begin{tabular}{cll} 
Layer & Magnitude ordering & $\Delta y$ increment \\
\hline I & $|\mathrm{MI}| \simeq|\mathrm{VF}| \gg|\mathrm{TI}|$ & $O\left(\nu / u_{\tau}\right)(\lesssim 3)$ \\
II & $|\mathrm{VF}| \simeq|\mathrm{TI}| \gg|\mathrm{MI}|$ & $O\left(\sqrt{\nu \delta / u_{\tau}}\right)(\simeq 1.6)$ \\
III & $|\mathrm{MI}| \simeq|\mathrm{VF}| \simeq|\mathrm{TI}|$ & $O\left(\sqrt{\nu \delta / u_{\tau}}\right) \quad(\simeq 1.0)$ \\
IV & $|\mathrm{MI}| \simeq|\mathrm{TI}| \gg|\mathrm{VF}|$ & $O(\delta)(\rightarrow 1)$
\end{tabular}

wall, $y^{+} \simeq 2.6 \sqrt{\delta^{+}}$, where the VF term loses leading order. This position is shown to have apparent relevance to a number of the statistics presented.

Overall, the aims of the present study are to document and describe statistical properties of zero pressure gradient boundary layers over the Reynolds number range $1,400 \leq \delta^{+} \leq 20,000$. An unusual (if not unique) attribute of the present measurements is that for any given free stream velocity, $U_{\infty}$, the present facility affords about a decade increase in Reynolds number (for $\delta^{+}>1,000$ ) by simply increasing the development fetch, $x$. Thus, for most of the primary data sets explored, the measurements were acquired in the same spatially developing boundary layer. Increasing Reynolds number in this manner removes possible dependencies on the in-flow boundary condition.

\section{Facility and Instrumentation}

\subsection{Flow Physics Facility}

The experiments were conducted in the Flow Physics Facility (FPF) at the University of New Hampshire (UNH). With test section dimensions of $\simeq 2.8 \mathrm{~m} \times 6 \mathrm{~m} \times$ $72 \mathrm{~m}$, the FPF is, to the authors' knowledge, the largest flow physics quality boundary layer wind tunnel in the world, e.g., see Klewicki (2010). The height of the test section is given approximately, since the upper wall diverges to account for displacement thickness growth. One advantage of the large flow development fetch is that it affords high Reynolds numbers at low speeds, and thus the measurements retain high spatial and temporal resolution.

In its current configuration (Phase I), the FPF is an open circuit suction tunnel that draws from, and discharges to, the atmosphere. The flow speed is driven by two $2.6 \mathrm{~m}$ diameter vane-axial fans, powered by two $300 \mathrm{~kW}$ AC motors. Speed control is attained by two ultra low-noise variable frequency drives, that are operated in a coupled leader-follower configuration. The maximum flow speed in the FPF test section is currently about $14.5 \mathrm{~m} / \mathrm{s}$. This corresponds to a volume flow rate of about $252 \mathrm{~m}^{3} / \mathrm{s}$. As described below, all of the measurements are for $U_{\infty} \gtrsim 7 \mathrm{~m} / \mathrm{s}$.

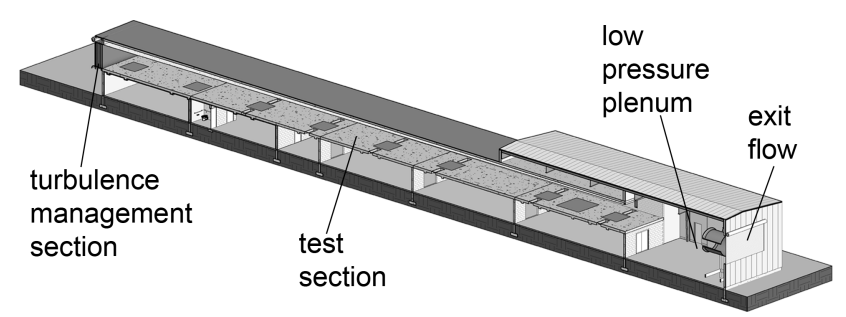

Fig. 1 Cut-away schematic drawing of the Flow Physics Facility. Flow is from left to right through the test section.

A cut-away schematic of the FPF is given in Fig. 1. Relative to this figure, the flow is from left to right, and is drawn through the test section by the pair of fans located in the low pressure plenum. The flow enters the test section through a constant area turbulence management section which consists of honeycomb followed by a series of five screens. Flow management of this kind has been previously demonstrated to work well at low speeds, and eliminates the possibility of generating Görtler vortices in the contraction (Klewicki \& Falco, 1990). The measurements were acquired in the boundary layer that develops along the floor of the FPF test section. The floor consists of three slabs of concrete separated by two expansion joints, at $x \simeq 25 \mathrm{~m}$ and $50 \mathrm{~m}$, respectively. The slabs are $25.4 \mathrm{~cm}$ thick, and were poured in place $\simeq 3 \mathrm{~m}$ above the ground plane using an extensive series of wooden frames. The mix of concrete was specially formulated to yield a dense microstructure. This promotes dimensional stability, and produces an unusually flat and smooth surface (when buffed). As verified by a professionally conducted survey, the deviations from perfectly flat are everywhere less than $0.26 \mathrm{~mm} / \mathrm{m}$. The entire surface was sealed and buffed. The current surface finish has not been quantified, but to the touch is comparable to a polished granite counter top. At the flow speeds investigated thus far, no effects of roughness have been observed in any of the measurements. The expansion joints present a surface variation of about $1 \mathrm{~mm}$ over $\simeq 3 \mathrm{~cm}$.

The boundary layers on the floor and sidewalls of the FPF are tripped immediately downstream of the turbulence management section. Hutchins (2012) discusses the recent study by Schlatter \& Orlu (2012) on issues pertaining to the effects of the trip on boundary layer development. Schlatter \& Orlu (2012) considered low $R_{\theta}\left(=U_{\infty} \theta / \nu\right)$ boundary layers, and used a body force tripping mechanism in their direct numerical simulations (DNS). They showed that if the trip is (1) in the boundary layer, (2) at sufficiently low Reynolds number and (3) does not over- or underexcite the boundary layer, then the artifacts of the tripping method should become undetectable by about $R_{\theta}=2,000$. The position of the trip in the FPF essen- 
tially assures (1) and (2), since, unlike tunnels with a contraction, the floor boundary layer in the FPF formally starts only about $1.4 \mathrm{~m}$ in front of the trip. The trip consists of a $6 \mathrm{~mm}$ threaded rod raised about $1 \mathrm{~mm}$ above the surface, i.e., so that there is flow both above and below the trip. Previous low speed studies found that this trip fixes the point of transition, and that the artifacts associated with the trip rapidly diminish downstream (Klewicki \& Falco, 1990). At the lowest $R_{\theta}$ of the present study $(\simeq 4230)$, no artifacts attributable to the trip are apparent.

Basic measures of flow quality are given by the free stream turbulence intensity and spanwise uniformity, and for the canonical flow, adherence to a zero pressure gradient. More specific measures relate to the properties of the mean and fluctuating velocity field. The axial free stream turbulence intensity in the FPF generally ranges between $0.2 \%$ and $0.5 \%$, depending on flow speed. Because the FPF draws from and exhausts to the atmosphere, gusty wind conditions can also influence the constancy of the FPF free stream flow at low speeds. In this case, low frequency variations in $U_{\infty}$ appear as an elevated turbulence intensity. The atmospheric wind conditions at the FPF are monitored by a pair of towermounted 3D sonic anemometers stationed outside and near the inlet. It is important to note, however, that except for storm-like conditions the effects of external gustiness are not detectable for flow speeds above about $3 \mathrm{~m} / \mathrm{s}$. For $U_{\infty} \geq 7 \mathrm{~m} / \mathrm{s}$ the streamwise free stream turbulence intensity, $u^{\prime} / U_{\infty}$, is less than about $0.3 \%$, where $u^{\prime}$ denotes rms.

Data pertaining to the constancy of $U_{\infty}$ as a function of downstream fetch are given in Fig. 2. This figure plots $U_{\infty}$ along the axial centerline of the FPF test section for three flow speeds, and also plots the percent variations from the given mean centerline speed. These data indicate that the present upper-wall contour results in a condition that closely approximates zero pressure gradient. At the higher speeds there is a distinct flow acceleration in the last three meters of the test section. This results from the abrupt area change into the downstream plenum.

Figure 3 shows Preston tube based friction velocity estimates across the central $5 \mathrm{~m}$ span at two streamwise locations for $U_{\infty} \simeq 7 \mathrm{~m} / \mathrm{s}$. The data in this figure were derived using the formula of Patel (1965). The linear curve-fits of these data demonstrate that the overall variation across the span is less than $0.5 \%$.

\subsection{Experiments and Instrumentation}

The experimental conditions and associated parameters are summarized in table 2 . The primary set of exper-

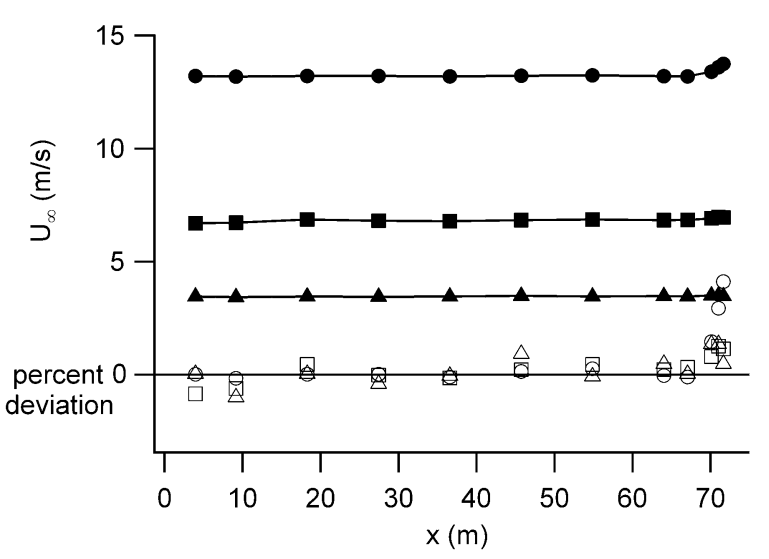

Fig. 2 Streamwise profiles of the FPF free stream velocity. Open symbols represent the percent deviation of the solid symbol profiles about their mean value.

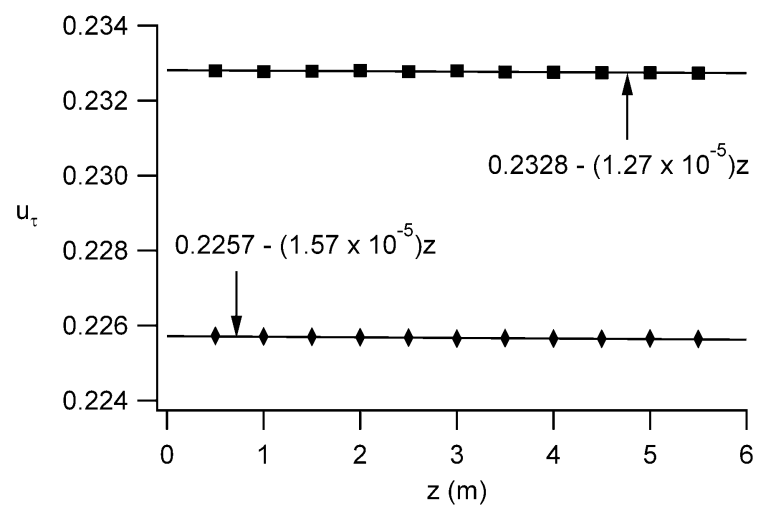

Fig. 3 Spanwise friction velocity surveys at $x=32 \mathrm{~m}$ and $64 \mathrm{~m}$ for $U_{\infty} \simeq 6.9 \mathrm{~m} / \mathrm{s}$.

iments are indicated by the open symbols, and these consisted of $U_{\infty} \simeq 7 \mathrm{~m} / \mathrm{s}$ boundary layer surveys at $x=$ $4 \mathrm{~m}, 8 \mathrm{~m}, 16 \mathrm{~m}, 32 \mathrm{~m}$ and $66 \mathrm{~m}$ from the trip, as well as $U_{\infty} \simeq 10.3 \mathrm{~m} / \mathrm{s}$ and $13.75 \mathrm{~m} / \mathrm{s}$ surveys at $x=66 \mathrm{~m}$. Table 2 also provides the parameters associated with a series of profiles at $U_{\infty} \simeq 10.5 \mathrm{~m} / \mathrm{s}$ (solid symbols). While constituting good quality measurements, some of these profiles do not extend as close to the wall as the open symbol data sets. They also more sparsely resolve the outer region when compared to those acquired at $7 \mathrm{~m} / \mathrm{s}$. This results in less accurate estimates of some of their integral parameters. They are, however, useful relative to documenting statistical properties in the interior portions of the flow, and thus are included as part of this investigation.

The profile measurements were acquired using a standard single wire hotwire sensor. The copper-plated $5 \mu \mathrm{m}$ tungsten wires contained within the probe are $3 \mathrm{~mm}$ in length with a $1 \mathrm{~mm}$ center active region. The copper plating allows the wires to be soft-soldered to the support prongs, and serves to aerodynamically isolate the 
Table 2 Properties of the FPF Boundary Layers. The sampling frequency is $f_{s}$, and $f_{c}^{+}$is the inner-normalized low-pass cut-off frequency. NF indicates that no filtering was applied. $H$ is the shape factor, $H=\delta^{*} / \theta$, where $\delta^{*}$ and $\theta$ are the mass and momentum deficit thicknesses, respectively. The sampling duration is $T$, and the inner-normalized sensor length is $\ell^{+}$.

\begin{tabular}{|c|c|c|c|c|c|c|c|c|c|c|c|}
\hline $\begin{array}{c}\text { data } \\
\text { symbol }\end{array}$ & $\begin{array}{c}x \\
(\mathrm{~m})\end{array}$ & $\begin{array}{c}U_{\infty} \\
(\mathrm{m} / \mathrm{s})\end{array}$ & $\begin{array}{c}u_{\tau} \\
(\mathrm{m} / \mathrm{s})\end{array}$ & $\begin{array}{l}\delta_{99} \\
(\mathrm{~m})\end{array}$ & $\begin{array}{c}\nu \\
\left(\mathrm{m}^{2} / \mathrm{s}\right)\end{array}$ & $\begin{array}{c}f_{s} \\
\left(1 / \mathrm{s} \times 10^{3}\right)\end{array}$ & $\delta^{+}$ & $H$ & $\ell^{+}$ & $f_{c}^{+}$ & $T U_{\infty} / \delta$ \\
\hline$\diamond$ & 4 & 6.87 & 0.263 & 0.0861 & $1.562 \times 10^{-5}$ & $10 \mathrm{kHz}$ & 1,450 & 1.352 & 16.8 & $\overline{0.86}$ & 23,940 \\
\hline$\triangleright$ & 8 & 6.95 & 0.252 & 0.1356 & $1.568 \times 10^{-5}$ & $10 \mathrm{kHz}$ & 2,180 & 1.355 & 16.1 & 0.94 & 15,370 \\
\hline$\triangleleft$ & 16 & 6.87 & 0.240 & 0.2456 & $1.505 \times 10^{-5}$ & $10 \mathrm{kHz}$ & 3,820 & 1.340 & 15.9 & 0.99 & 8,390 \\
\hline$\nabla$ & 32 & 7.01 & 0.234 & 0.4284 & $1.559 \times 10^{-5}$ & $10 \mathrm{kHz}$ & 6,430 & 1.310 & 15.0 & 1.08 & 3,920 \\
\hline$\Delta$ & 66 & 6.95 & 0.226 & 0.7363 & $1.545 \times 10^{-5}$ & $10 \mathrm{kHz}$ & 10,770 & 1.290 & 14.6 & 2.48 & 2,260 \\
\hline$\bullet$ & 4 & 10.43 & 0.397 & 0.0838 & $1.555 \times 10^{-5}$ & $20 \mathrm{kHz}$ & 2,280 & 1.296 & 25.5 & $\mathrm{NF}$ & 3,730 \\
\hline$\triangleright$ & 8 & 10.52 & 0.374 & 0.1340 & $1.533 \times 10^{-5}$ & $20 \mathrm{kHz}$ & 3,270 & 1.301 & 24.4 & $\mathrm{NF}$ & 3,530 \\
\hline 4 & 16 & 10.63 & 0.351 & 0.2336 & $1.455 \times 10^{-5}$ & $10 \mathrm{kHz}$ & 5,680 & 1.311 & 24.1 & $\mathrm{NF}$ & 3,640 \\
\hline $\boldsymbol{\nabla}$ & 32 & 10.72 & 0.345 & 0.3953 & $1.521 \times 10^{-5}$ & $10 \mathrm{kHz}$ & 8,970 & 1.308 & 22.7 & $\mathrm{NF}$ & 3,250 \\
\hline$\Delta$ & 64 & 10.21 & 0.327 & 0.7439 & $1.571 \times 10^{-5}$ & $10 \mathrm{kHz}$ & 15,480 & 1.265 & 20.8 & $\mathrm{NF}$ & 2,470 \\
\hline$\square$ & 66 & 10.33 & 0.330 & 0.7171 & $1.503 \times 10^{-5}$ & $10 \mathrm{kHz}$ & 15,740 & 1.252 & 22.0 & 0.86 & 3,450 \\
\hline 0 & 66 & 13.75 & 0.432 & 0.6885 & $1.512 \times 10^{-5}$ & $20 \mathrm{kHz}$ & 19,670 & 1.258 & 28.6 & $\mathrm{NF}$ & 5,990 \\
\hline
\end{tabular}

center sensing region. The probes were operated using AA Lab Systems AN-1004 anemometers at heating ratios between 1.5 and 1.7. The frequency response of the sensor-anemometer system was estimated using the impulse response module on the anemometer, and always exceeded $15 \mathrm{kHz}$. This was always significantly beyond the frequencies of the turbulence in the present boundary layers. Pre- and post-calibrations were performed in the free stream flow, and the experiment was discarded if these calibrations did not agree to within about $\pm 1 \%$. The velocities for the calibrations were derived from a Pitot-static tube connected to an MKS-Baratron 10 torr differential pressure transducer. The barometric pressure was monitored throughout each experiment. Temperature data were measured with a thermocouple at each $x$ station, and were collected in concert with the freestream dynamic pressure and hotwire time series. Temperature variations during any given experiment were generally less than $\pm 1.5^{\circ} \mathrm{C}$.

The hotwire data were sampled at the rates indicated in Table 2 using a National Instruments PXI-6221 16 bit analog to digital converter. As shown in Table 2 most of the signals from the primary data sets were analog filtered at the indicated cut-off frequencies prior to digitization. No analog filtering was applied to the other signals. The influence of this was investigated in post-processing by applying a low pass digital filter at $f_{c}^{+} \simeq 1$. This caused no detectable change from the unfiltered results for statistics up to kurtosis, $K(u)$. The sampling durations generally varied depending on $x$ and $U_{\infty}$. The competing considerations here were to obtain good statistical convergence while keeping the experiment duration short enough to avoid issues such as calibration drift and significant temperature variations. According to the estimates of Klewicki \& Falco (1990), the present sampling durations should yield about $\pm 5 \%$ convergence uncertainty in the skewness of $u, S(u)$, and less uncertainty for the mean, variance and kurtosis. The profiles were obtained using a stepper motor driven Velmex $1.4 \mathrm{~m}$ traverse fitted with a custom-built aerodynamic shroud. The probe was attached to the end of a sting that extended $0.5 \mathrm{~m}$ upstream of the traverse.

The friction velocity was estimated using a Clauser chart with the logarithmic profile coefficients (in Eq. 3 below) of $\kappa=0.384$ and $B=4.17$ of Monkewitz, Chauhan \& Nagib (2008). For the zero pressure gradient flow, Nagib, Chauhan \& Monkewitz (2007) demonstrate that oil film interferometry based measurements of skin friction coefficient exhibit excellent agreement $( \pm 1.5 \%)$ with the Coles-Fernholz relation employing $\kappa=0.384$ and $C=4.127$,

$C_{f}=2\left[\kappa^{-1} \ln \left(\mathrm{R}_{\theta}\right)+\mathrm{C}\right]^{-2}$.

Figure 4 compares the present estimates of $C_{f}$ with those determined using Eq. 2. To provide a sense of the deviation from Eq. $2, \pm 5 \%$ error bars are included. These results indicate that the present $u_{\tau}$ estimates are within $\pm 2.5 \%$ of the $u_{\tau}$ values determined using Eq. 2. For the flat plate flow, this agreement between the Clauser plot and Eq. 2 is anticipated since both are directly based upon the properties of the logarithmic mean velocity profile. For reference, the oil-film based estimates of $u_{\tau}$ reported by Nagib, Chauhan \& Monkewitz (2007) have a scatter that is slightly greater than $\pm 1 \%$ about the $u_{\tau}$ curve determined using Eq. 2 .

\section{Results}

Mean profile properties are examined first, followed by the statistics of the turbulent fluctuations. The data 


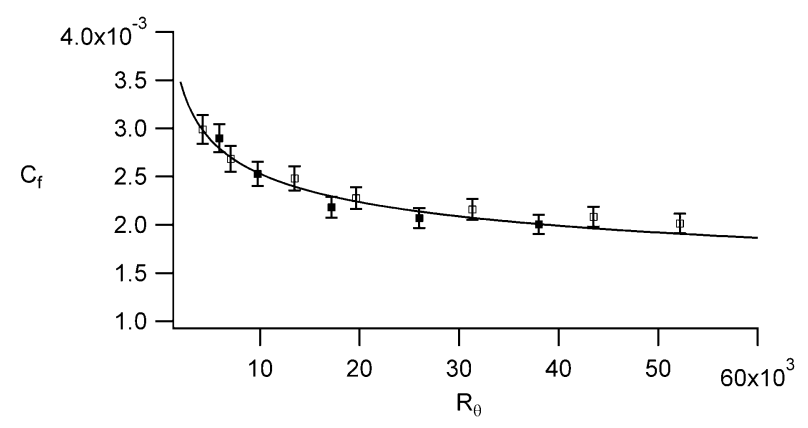

Fig. 4 Clauser plot based $u_{\tau}$ estimates from the present experiments. Open and solid symbols are from the primary and secondary data sets described in table 2. Solid line is Eq. 2.
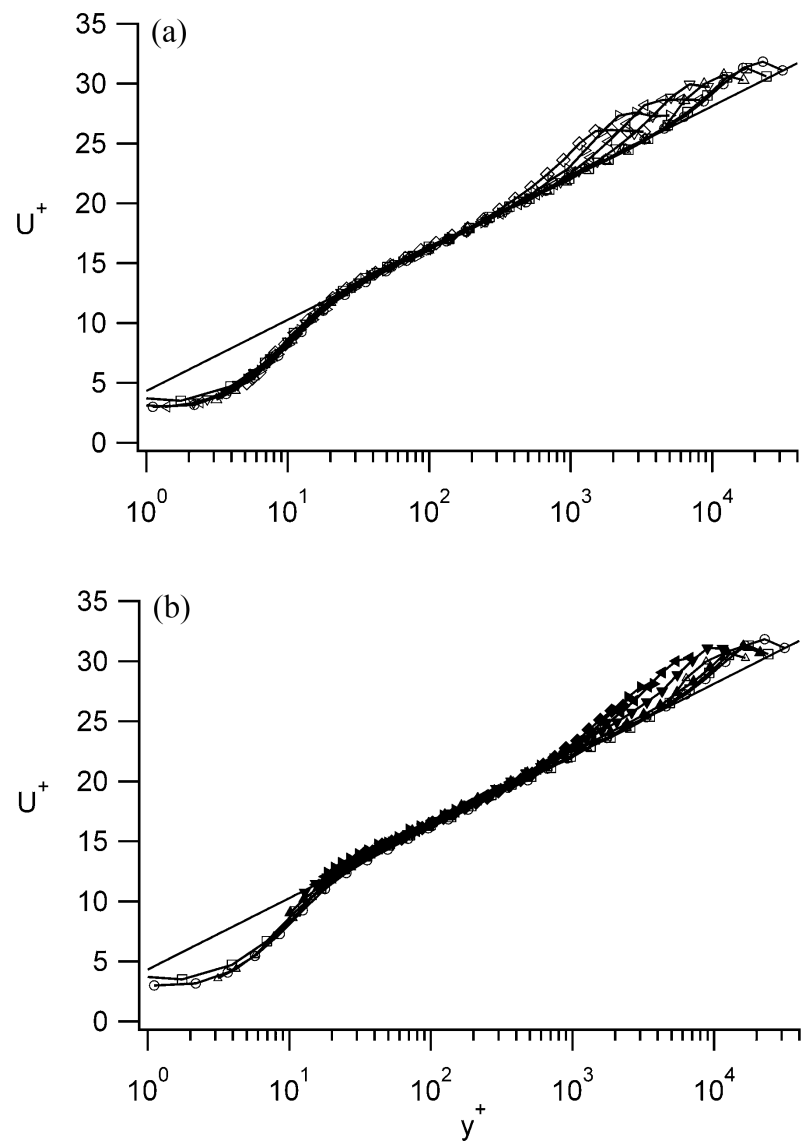

Fig. 5 Logarithmic mean profiles; (a) $U_{\infty} \simeq 7 \mathrm{~m} / \mathrm{s}$ and $U_{\infty}=10.33 \mathrm{~m} / \mathrm{s}$ and $13.75 \mathrm{~m} / \mathrm{s}$ profiles at $66 \mathrm{~m}$, and $(\mathrm{b}) U_{\infty} \simeq$ $10.5 \mathrm{~m} / \mathrm{s}$ compared to the $U_{\infty}=10.33 \mathrm{~m} / \mathrm{s}$ and $13.75 \mathrm{~m} / \mathrm{s}$ profiles at $66 \mathrm{~m}$. Symbols are given in Table 2 . Solid line is given by $U^{+}=0.387^{-1} \ln \left(\mathrm{y}^{+}\right)+4.32$ (Marusic et al., 2013).

presentation closes with a brief examination of some of the spectral properties associated with $u(t)$.

\subsection{Mean Velocity Profile Properties}

Mean velocity profile data are plotted on semi-logarithmic axes in Figs. 5a,b. The open symbols in Table 2 are shown in Fig. 5a, while the closed symbol data and the higher $\delta^{+}$data from the primary data sets are shown in Fig. 5b. All of the profiles of Fig. 5a exhibit very good agreement with the boundary layer logarithmic profile given by Marusic et al. (2013), and in particular adhere closely to their formula $U^{+}=(0.387)^{-1} \ln \left(\mathrm{y}^{+}\right)+4.32$. (The data exhibit similarly good agreement using the values of Monkewitz, Chauhan \& Nagib (2008) for $\kappa$ and $B$ noted above.) These data also self-consistently exhibit good agreement with each other down to near $y^{+} \simeq 3$. The profiles of Fig. 5b display similarly good agreement. Small deviations from the primary data sets are that a couple of the profiles exhibit a slightly stronger wake, see below, and the lowest $\delta^{+}$profile shows a slightly more pronounced upward deviation from the logarithmic line in the region $y^{+}<100$. Most of the present profiles exhibit a small but discernible overshoot in streamwise velocity at the very outer edge of the boundary layer. The origin of this is presently unknown.

The behavior of the logarithmic region is an ongoing topic of considerable interest, e.g., Marusic et al. (2010); Klewicki (2010). In this regard, Marusic et al. (2013) provide evidence that, as the $\delta^{+}$gets large, the inverse of the leading coefficient in the logarithmic law,

$U^{+}=\kappa^{-1} \ln \left(\mathrm{y}^{+}\right)+\mathrm{B}$,

i.e, von Kármán's constant, approaches the same constant value $(0.38 \leq \kappa \leq 0.39)$ for smooth-wall turbulent pipe and boundary layer flows. Their analysis also provides evidence that the region of emerging logarithmic dependence has a lower boundary that scales with $\sqrt{\delta^{+}}$, and an upper boundary that scales with $\delta^{+}$. This is in accord with recent analyses of the governing mean equation (Fife et al., 2005, 2009; Klewicki, Fife \& Wei, 2009; Klewicki, 2013a).

Per the analysis of Marusic et al. (2013), Fig. 6 plots the deviation of the velocity profile from $\kappa^{-1} \ln \left(\mathrm{y}^{+}\right)$. Here we use their boundary layer value of $\kappa=0.387$, while the horizontal line indicates the associated value of $B=4.32$. Fig. 6 a plots the present $\delta^{+}=6,430,10,770$, and 19,670 profiles versus $y^{+}$, and Fig. $6 \mathrm{~b}$ shows these same data versus $y / \delta$. Both figures also present the $\delta^{+}=15,670$ profile of Kulandaivelu (2012). Note that for the purposes of self-consistent comparison, all $\delta^{+}$ values are based upon $\delta_{99}$. A distinctive Reynolds number trend indicated by the present data is that the nearwall bump in the profile is quite pronounced and rises above the logarithmic line at low $\delta^{+}$. With increasing $\delta^{+}$, the bump becomes less pronounced (flattens) and the entire profile shifts downward such that the logarithmic line is approached from below at high $\delta^{+}$, e.g., 

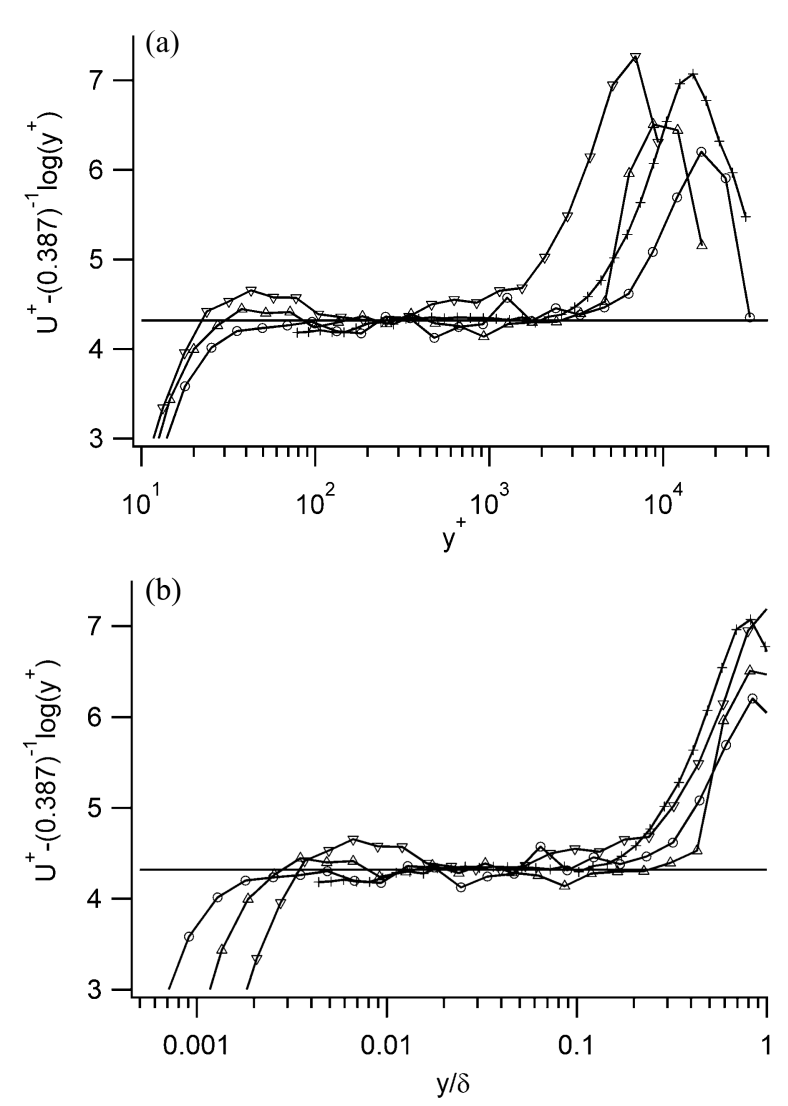

Fig. 6 Deviation of the measured mean profiles from $U^{+}=$ $0.387^{-1} \ln \left(\mathrm{y}^{+}\right) ;(\mathrm{a})$ versus $y^{+},(\mathrm{b})$ versus $y / \delta$. Closed symbols are the $\delta^{+}=6,430,10,770$, and 19,670 described in Table 2 . Plus symbols denote the $\delta^{+}=15670$ data of Kulandaivelu (2012).

compare the present $\delta^{+}=19,670$ profile with that of Kulandaivelu. The overall $\delta^{+}$trend is supported by the profiles of Kulandaivelu (2012), while the approach to logarithmic behavior from below is also apparent in the pipe flow data analyzed in Marusic et al. (2013).

From the data of Figs. 6a,b, it is rational to surmise that the wall-normal position where the region of logarithmic dependence begins scales on a variable that is intermediate to $\nu / u_{\tau}$ and $\delta$. This is in accord with analyses of the mean dynamical equation, e.g., Klewicki (2013a). Specifically, these analyses identify two regions where the mean momentum equation admits self-similar behavior as $\delta^{+} \rightarrow \infty$. One region extends from $y^{+} \simeq 2.6 \sqrt{\delta^{+}}$to $y^{+}=C \delta^{+}$, where $C \lesssim 0.5$. This is the domain where the invariant form of the mean equation admits a logarithmic mean profile solution. The other domain is bounded by $y^{+} \simeq 7$ and $y^{+} / \sqrt{\delta^{+}} \simeq 2.6$, and thus contains the mean profile bump note above. The analyses indicate that the flow on the outer domain will approach exact self-similarity more rapidly than on the inner domain, which seems

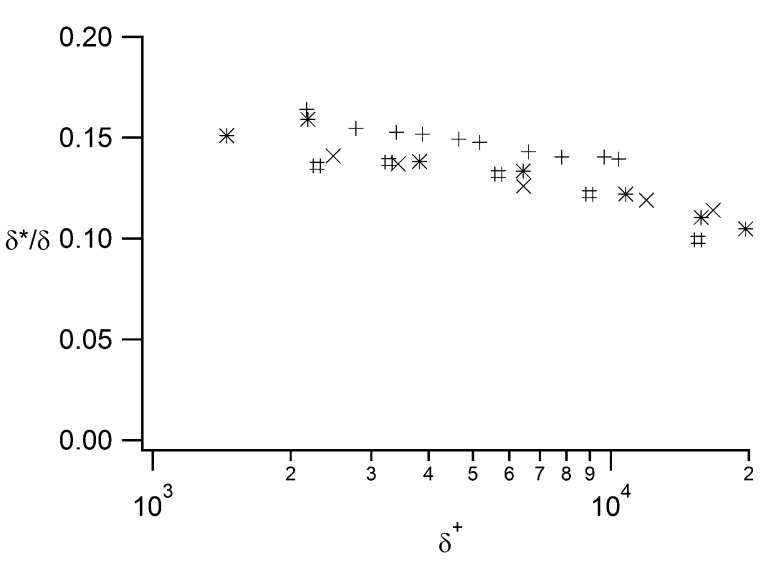

Fig. 7 Outer-normalized displacement thickness versus $\delta^{+}$; primary FPF data sets (open symbols Table 2), $* ; U_{\infty}=$ $10.5 \mathrm{~m} / \mathrm{s}$ FPF data sets (solid symbols Table 2), \#; Hutchins et al. (2009), ×; Kulandaivelu (2012), +.

to be supported by the present observations. The evolution of the bump is associated with the scale separation between the near-wall region where the characteristic length scale is $O\left(\nu / u_{\tau}\right)$ and the region where the mean viscous force loses leading order. This region has a characteristic length that is $O\left(\sqrt{\nu \delta / u_{\tau}}\right)$, see Table 1 above, and Fig. 11 below.

Other properties associated with the profiles of Fig. 5 are shown in Figs. 7 - 10. On these figures we also compare with the results of Hutchins et al. (2009) and $\mathrm{Ku}-$ landaivelu (2012). Figures 7 - 9 respectively show the mass deficit thickness, $\delta^{*}$, the momentum deficit thickness, $\theta$, and their ratio the shape factor, $H=\delta^{*} / \theta$. For consistency of comparison, all of these quantities were determined using profile integrations from the wall to $\delta=\delta_{99}$. As indicated, the present $\delta^{*} / \delta$ and $\theta / \delta$ measurements from the FPF are in good agreement with those from the HRNBLWT. Note, however, that for the reasons discussed previously the $U_{\infty}=10.5 \mathrm{~m} / \mathrm{s}$ data (derived from the solid symbol experiments in table 2) exhibit slightly more scatter than the open symbol experiments. It is also noted that the data scatter in Figs. 7 and 8 is larger than in the Fig. 9. This indicates the existence of slight deviations in the absolute values of $\delta^{*} / \delta$ and $\theta / \delta$ from experiment to experiment, and is likely to connected to the thickness of the boundary layer at the trip. Collectively, however, the $H=\delta^{*} / \theta$ values provide convincing evidence that beyond an initial offset relative to $\delta$ at the trip, $\delta^{*}$ and $\theta$ develop self-similarly in each of these flows.

Coles (1956) wake strength parameter is given by $\Pi=\kappa \Delta U^{+} / 2$, where $\Delta U^{+}$is the maximum deviation from the logarithmic line (3). For the data of Fig. 10, $\Pi$ was computed using the coefficients of the logarithmic line of Fig. 5. Overall, the present FPF results show 


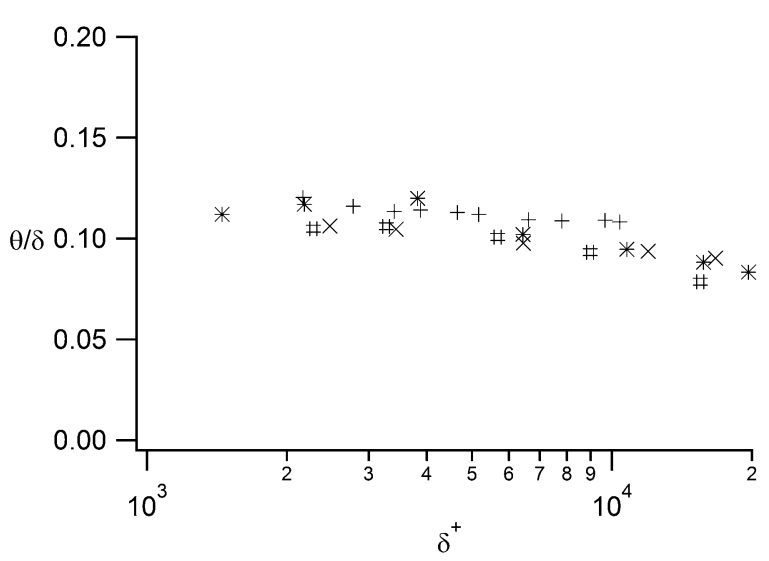

Fig. 8 Outer-normalized momentum thickness versus $\delta^{+}$; symbols same as in Fig. 7.

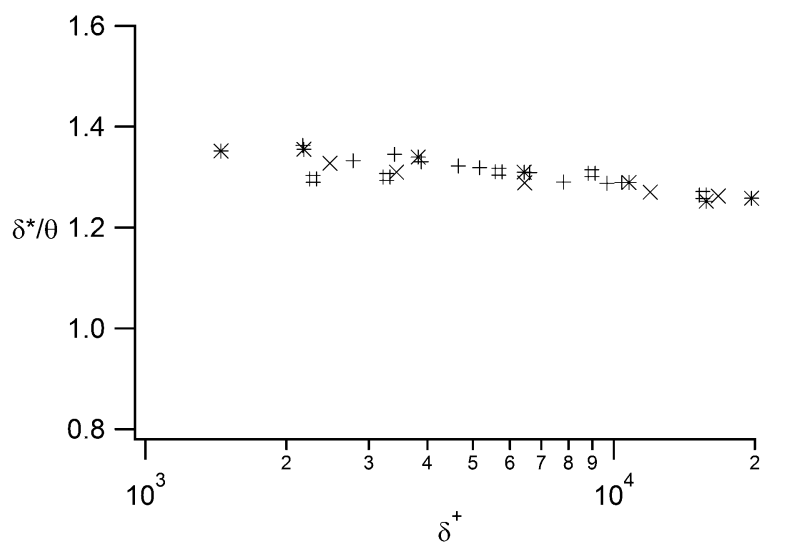

Fig. 9 Shape factor versus $\delta^{+}$; symbols same as in Fig. 7.

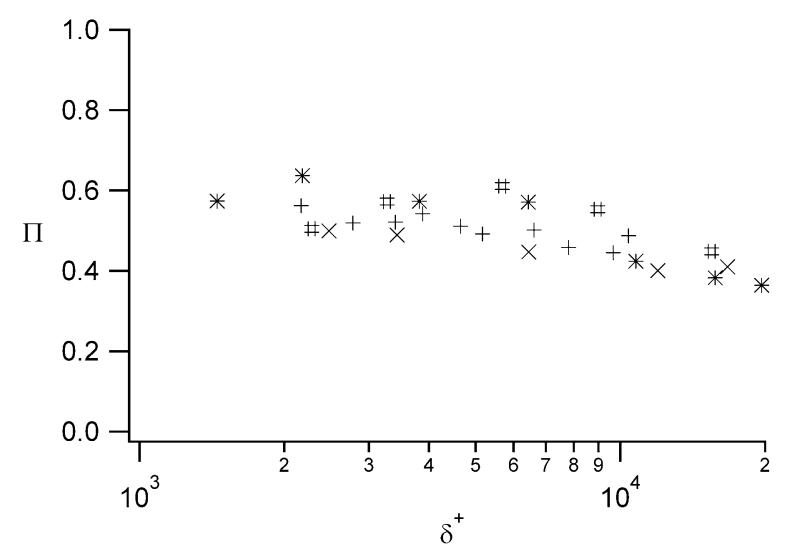

Fig. 10 Wake strength parameter $\Pi$ versus $\delta^{+}$; symbols same as in Fig. 7.

good agreement with those from the HRNBLWT, but in general exhibit slightly higher values. Both the FPF and HRNBLWT data indicate that $\Pi$ exhibits a decreasing trend with increasing $\delta^{+}$for $\delta^{+}$greater than about 5,000 .

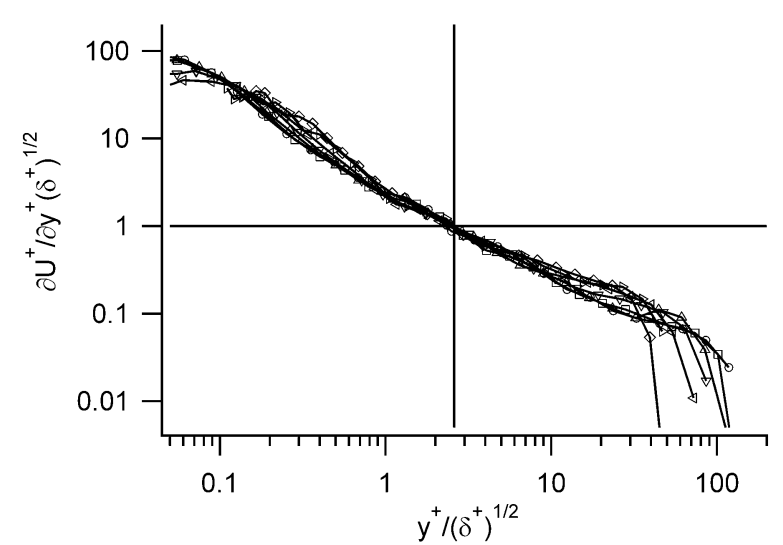

Fig. 11 Mean velocity gradient profiles from the primary data sets normalized using the intermediate length $\sqrt{\nu \delta / u_{\tau}}$; symbols given in Table 2. Vertical line denotes $y^{+} / \sqrt{\delta^{+}}=$ 2.6 .

Multiscale analysis of the mean momentum equation indicates that $\partial U^{+} / \partial y^{+}=O\left(1 / \sqrt{\delta^{+}}\right)$at $y^{+}=$ $O\left(\sqrt{\delta^{+}}\right)$, for all Reynolds numbers beyond the transitional regime (Fife et al., 2005, 2009). These behaviors are associated with the invariant form admitted by the momentum equation, and the similarity solution that results (Klewicki, 2013a). This analytically predicted behavior is empirically explored in Fig. 11 by plotting $\sqrt{\delta^{+}} \partial U^{+} / \partial y^{+}$versus $y^{+} / \sqrt{\delta^{+}}$. An extensive analysis of existing turbulent pipe, channel and boundary layer flows reveals that $\sqrt{\delta^{+}} \partial U^{+} / \partial y^{+}$equals unity at $y^{+} / \sqrt{\delta^{+}} \simeq 2.6$ (Klewicki, 2013b). Physically, this corresponds to the location where the mean viscous force in the momentum equation loses leading order, see Table 1. Consistent with the findings of Marusic et al. (2013) and the data of Figs. 5 and 6, this location is also where the mean velocity profile is predicted to begin to exhibit the similarity solution associated with logarithmic behavior (Klewicki, 2013a). In accord with analyses at lower $\delta^{+}$(Klewicki, 2013b), the present $\sqrt{\delta^{+}} \partial U^{+} / \partial y^{+}$profiles pass through unity at $y^{+} / \sqrt{\delta^{+}} \simeq 2.55$.

\subsection{Statistics of the Streamwise Velocity Fluctuations}

Profiles of the inner normalized streamwise velocity variances are given in Figs. 12a,b for the primary and secondary data sets. Per previous plots, the secondary data are compared with the two highest $\delta^{+}$profiles from the primary experiments. The two data sets exhibit a number of consistently held features. One is a peak value near $y^{+}=15$ that, under the present normalization, increases with increasing $\delta^{+}$(Klewicki \& Falco, 1990; DeGraaff \& Eaton, 2000; Metzger \& Klewicki, 2001; Hutchins et al., 2009). A second is an emerg- 

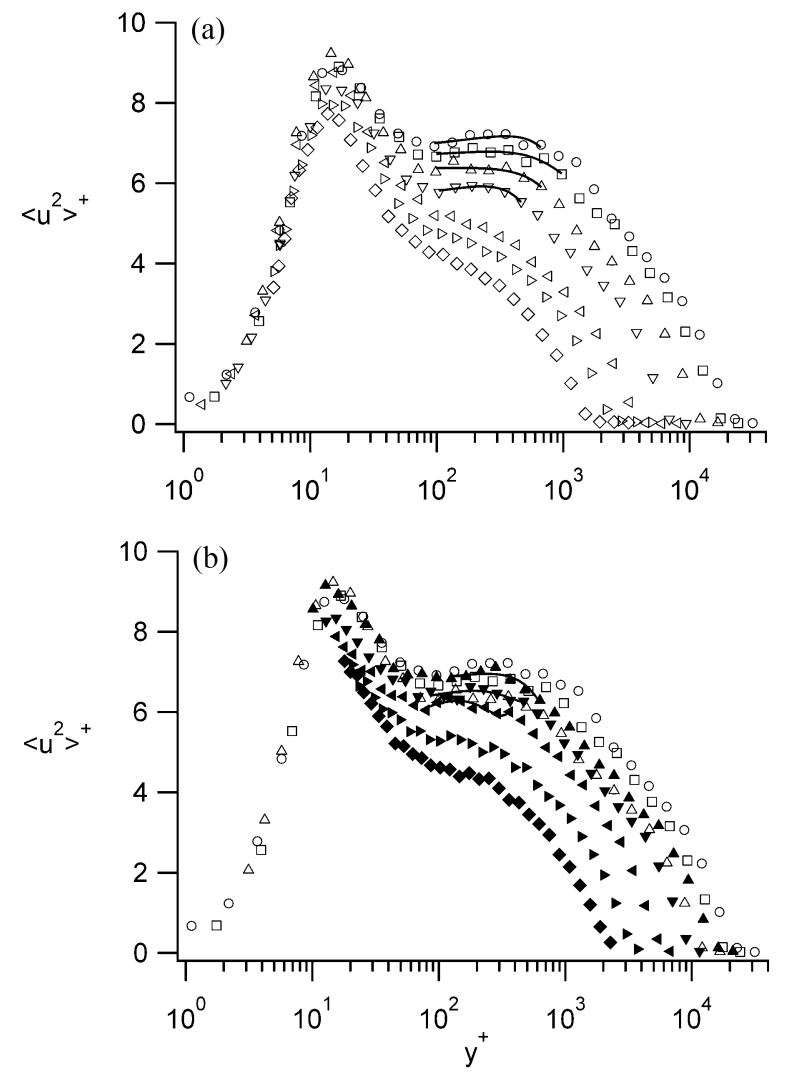

Fig. 12 Inner normalized profiles of the streamwise velocity variance; (a) $U_{\infty} \simeq 7 \mathrm{~m} / \mathrm{s}$ and $U_{\infty}=10.33 \mathrm{~m} / \mathrm{s}$ and $13.75 \mathrm{~m} / \mathrm{s}$ profiles at $66 \mathrm{~m}$, (b) $U_{\infty} \simeq 10.5 \mathrm{~m} / \mathrm{s}$ compared to the $U_{\infty}=$ $10.33 \mathrm{~m} / \mathrm{s}$ and $13.75 \mathrm{~m} / \mathrm{s}$ profiles at $66 \mathrm{~m}$. Symbols are given in Table 2 .

ing peak near $y^{+}=2.6 \sqrt{\delta^{+}}$that also has increasing inner-normalized amplitude with increasing $\delta^{+}$. While the emergence of a spectral peak at this location has been documented for some time, e.g., Hutchins \& Marusic (2007b), the emergence of a peak in the broadband statistic is less clear, e.g., Priyadarshana et al. (2007); Hutchins et al. (2009). The polynomial curve-fits on the Figs. 12a,b are used below to characterize the position and magnitude of this apparently emerging feature. A third feature is the emergence of a region of logarithmic decay in $\left\langle u^{2}\right\rangle^{+}$on a domain starting near to, but outward of, $y^{+}=2.6 \sqrt{\delta^{+}}$. Marusic et al. (2013) provide empirical evidence that this domain coincides with where the logarithmic $U^{+}\left(y^{+}\right)$profile also exists. The three features just noted are now explored.

The position of the emerging mid-layer peak, $y_{m i d}^{+}$, in the $\left\langle u^{2}\right\rangle^{+}$profile was estimated as a function of $\delta^{+}$ using the curve-fits shown in Figs. 12a,b. These are shown in Fig. 13. The amplitudes of the mid-layer peaks were similarly estimated, and these are plotted along with the measured near-wall peak values in Fig. 14. The data of Fig. 13 indicate that for the present data

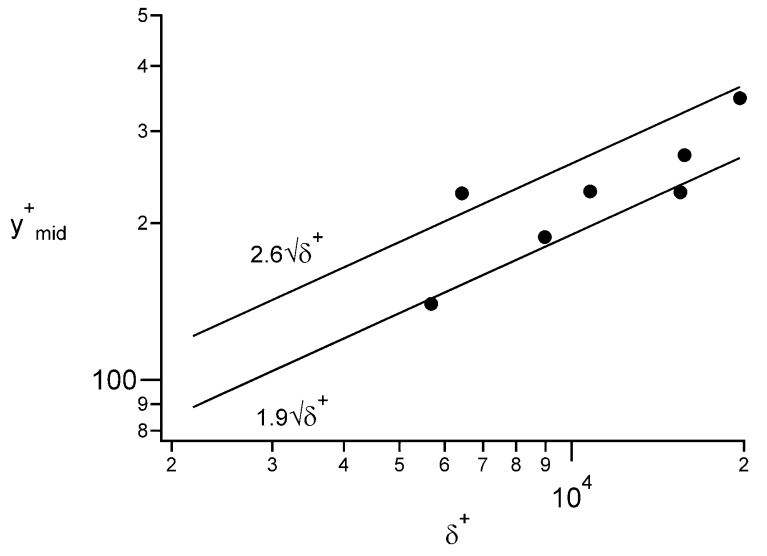

Fig. 13 Estimated position of the emerging mid-layer peak of $\left\langle u^{2}\right\rangle^{+}$versus $\delta^{+}$.

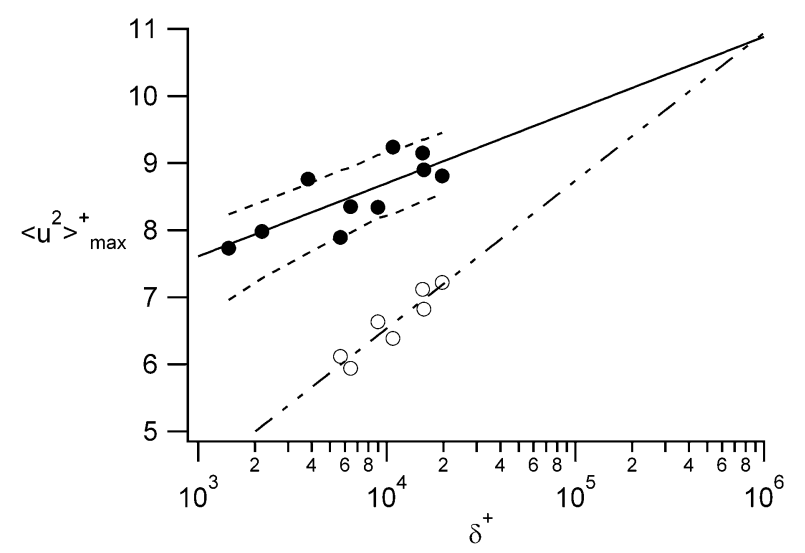

Fig. 14 Maximum values of the $\left\langle u^{2}\right\rangle^{+}$peaks versus $\delta^{+}$; nearwall peak values, $\bullet$; mid-layer peak values, $\bigcirc$. Curve-fits given by $1.07 \log \left(\delta^{+}\right)+4.4, \longrightarrow ; 2.2 \log \left(\delta^{+}\right)-2.28,--\ldots$; dashed lines indicate the near-wall peak values for a sensor of $\ell^{+}=0$ (upper curve) and $\ell^{+}=25$ (lower curve), as estimated using the formula of Hutchins et al. (2009).

sets the mid-layer peak nominally occurs in the region $1.9 \sqrt{\delta^{+}} \lesssim y^{+} \lesssim 2.6 \sqrt{\delta^{+}}$. This is in the outer portion of the third layer associated with the mean momentum balance (Wei et al., 2005). In this layer all three terms in that equation are of leading order importance, see Table 1 . The location $y^{+} \simeq 1.9 \sqrt{\delta^{+}}$coincides with the zero-crossing of the mean effect of turbulent inertia, while $y^{+} \simeq 2.6 \sqrt{\delta^{+}}$is where the mean viscous force loses leading order importance, i.e., is at the boundary between force balance layer III and IV. Physically, $y_{\text {mid }}^{+}$ appears to track the interface just interior to where the inertially dominated momentum sink-like motions, on average, first appear (Klewicki et al., 2007).

The near-wall peak amplitudes plotted in Fig. 14 adhere closely to the logarithmic trend revealed by the previous high resolution studies discussed in the Introduction. In addition, the curve-fit of the present data 


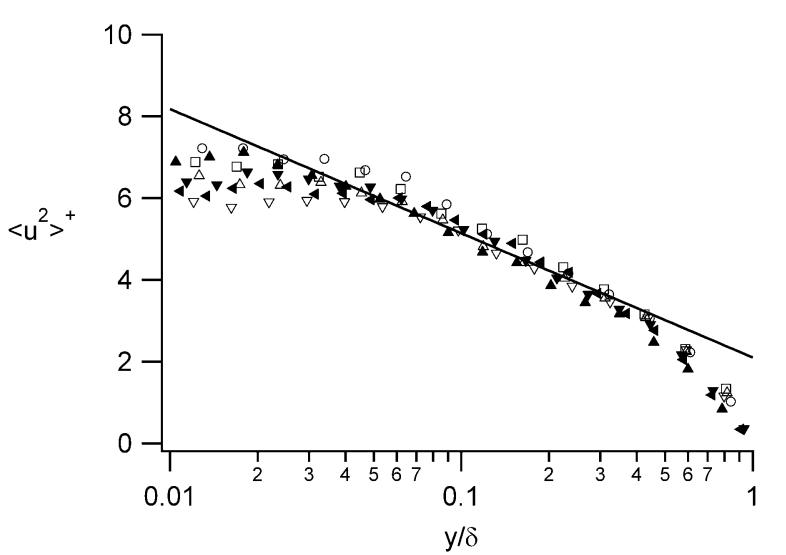

Fig. 15 Profiles of the streamwise velocity variance plotted versus $y / \delta$ for $\delta^{+} \gtrsim 5,000$. Line is given by $\left\langle u^{2}\right\rangle^{+}=$ $-1.32 \ln (\mathrm{y} / \delta)+2.1$. Symbols are given in Table 2 .

convincingly bisects the upper and lower bounds estimated by the formula of Hutchins et al. (2009) for an $\ell^{+}$equal to 0 and 25 , respectively. These bounds are indicated by the upper and lower dashed curves on the figure. Note, however, that no corrections were applied to the present data.

Like the near-wall peak value, the estimates for the mid-layer peak also exhibit an approximately logarithmic dependence on $\delta^{+}$, but with a larger slope. Given this, extrapolation of the two curve-fits leads to the expectation that the mid-layer peak will exceed the near-wall peak at $\delta^{+} \simeq 900,000$. Data acquired at the SLTEST site in Utah's west desert at $\delta^{+} \simeq 1,000,000$ provide evidence of an outer peak value near $y^{+} \simeq$ $2.6 \sqrt{\delta^{+}}$, but the near-wall peak value was measured to be somewhat larger, and the value for the mid-layer peak was measured to be somewhat smaller, than estimated in Fig. 14 (Priyadarshana et al., 2007; MorrillWinter \& Klewicki, 2013). The SLTEST site measurements also indicate that at $\delta^{+} \simeq 1,000,000$ the midlayer peak value is still less than the near-wall peak. As demonstrated by Pullin et al. (2013), the recent pipe flow measurements of Hultmark et al. (2012) indicate an approximately logarithmic increase in the mid-layer peak value, but the rate of increase exhibited by their data is slightly less than suggested by the present data.

Figure 15 pertains to the logarithmic decay of $\left\langle u^{2}\right\rangle^{+}$ with increasing $y$ on the inertial domain beginning beyond $y^{+} \simeq 2.6 \sqrt{\delta^{+}}$. Per Townsend's attached eddy hypothesis, Fig. 15 plots $\left\langle u^{2}\right\rangle^{+}$versus $y / \delta$ for the present $\delta^{+} \gtrsim 5,000$ data. As indicated, the present measurements provide evidence of the predicted logarithmic decay on the relevant domain, although there is some scatter in data. The indicated fit is given by $\left\langle u^{2}\right\rangle^{+}=$ $-1.32 \ln (\mathrm{y} / \delta)+2.1$. Both the leading coefficient and the additive constant are within the uncertainty bounds of

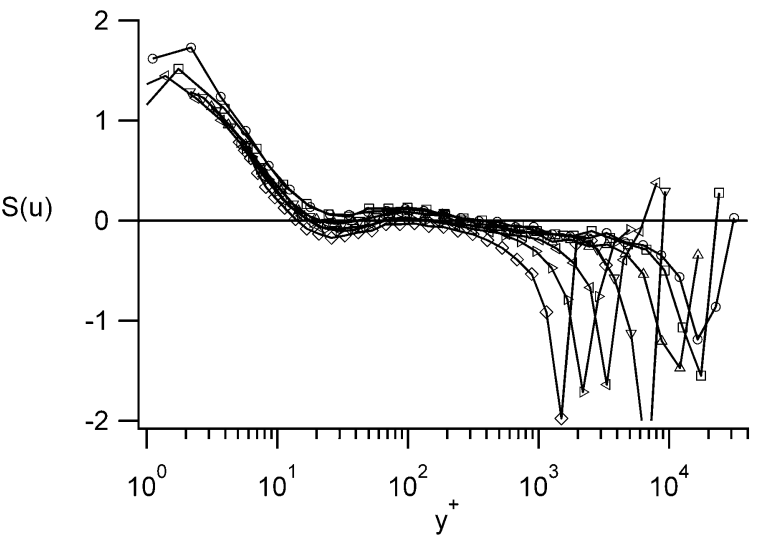

Fig. 16 Profiles of the streamwise velocity skewness plotted versus $y^{+}$for the primary data sets. Symbols are given in Table 2 .

the HRNBLWT boundary layer results of Marusic et al. $(2013),\left\langle u^{2}\right\rangle^{+}=-1.26 \ln (\mathrm{y} / \delta)+2.3$. Part of the observed difference is associated with their use of a boundary layer thickness estimate that is somewhat larger than $\delta_{99}$ used in the present study.

Profiles of the skewness of the probability density function of $u, S(u)$, are shown in Fig. 16 for the primary data sets. Here we focus on a couple of features. Consistent with previous observations, the negative portion of the $S(u)$ profile in the near-wall region shifts upward with increasing $\delta^{+}$. From their measurements at the SLTEST site, Metzger \& Klewicki (2001) showed that at sufficiently large $\delta^{+}$this upward shift is such that $S(u)$ eventually becomes entirely positive in this region of the flow. They further showed that this upward shift stems from the nearly additive influence of increasingly low frequency motions with increasing $\delta^{+}$. Mathis et al. (2009) subsequently showed that this low frequency effect is associated with the modulation of the near-wall flow by larger scale motions (so-called superstructures) whose primary signature is the emerging mid-layer peak in $\left\langle u^{2}\right\rangle^{+}$discussed above. They showed that the correlation coefficient describing this modulation effect exhibits behaviors similar to that of the $S(u)$ profile, and Mathis et al. (2012) later showed that this is directly related to the contributions to $S(u)$ associated with the nonlinear coupling between the large and small scale motions.

Here we explore connections between $S(u)$, the modulation correlation coefficient, and the mid-layer peak in $\left\langle u^{2}\right\rangle^{+}$by quantifying the outer (mid-layer) zero-crossing of $S(u)$. In this regard, the Reynolds number must be sufficient for $S(u)$ to become positive beyond the nearwall region, see Fig. 16. Figure 17 shows the mid-layer zero-crossing, $y_{0}^{+}$, for all of the present $S(u)$ data having $\delta^{+} \gtrsim 2,000$. The zero-crossing position was esti- 


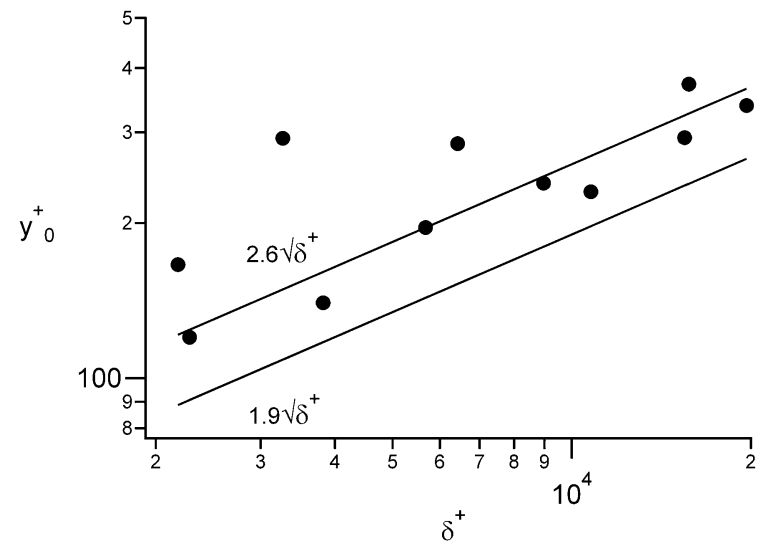

Fig. 17 Mid-layer zero crossing of $S(u)$ versus $\delta^{+}$.

mated using a simple linear interpolation between the data points straddling $S(u)=0$. Owing to the shallow downward trajectory of the profile, this estimate was quite sensitive to data scatter, and especially so at low $\delta^{+}$. This is reflected in the data of Fig. 17. With increasing $\delta^{+}$, however, the zero-crossing data scatter about $y^{+} \simeq 2.6 \sqrt{\delta^{+}}$. This finding agrees with the results of Mathis et al. (2009) showing that the zerocrossing of the modulation correlation coefficient also adheres closely to $y^{+} \simeq 2.6 \sqrt{\delta^{+}}$. Similarly, comparison with Fig. 13 reveals that the zero-crossing of $S(u)$ coincides closely with the position of the mid-layer peak in $\left\langle u^{2}\right\rangle^{+}$. This position is physically where the viscous force in the mean momentum equation loses leading order (Wei et al., 2005), and just beyond where the turbulent inertia term in this equation (when viewed as a force) shifts from a momentum source to momentum sink (Klewicki et al., 2007).

Figure 18 shows profiles of the kurtosis of the $u$ fluctuations, $K(u)$, for the primary data sets. These profiles exhibit a near-wall crossing of the $K(u)=3$ line (the Gaussian value), but with an indication of a slight Reynolds number dependence in position and the magnitude of the maximum excursion below 3 near $y^{+}=15$. Across the interior of the flow, $K(u)$ is slightly subGaussian, with the upward crossing through $K(u)=3$ consistently occurring near $y / \delta=0.5$ for all of the $\delta^{+}$values explored. Consistent with increasingly intermittent signals, $K(u)$ attains large values both in the viscous sublayer and near $y=\delta$. The magnitude of $K(u)$ in the viscous sublayer appears to exhibit an increasing trend with increasing Reynolds number. This observation is consistent with previous measurements in the near-neutral atmospheric surface layer (Metzger \& Klewicki, 2001), but requires further investigation. Interestingly, the large amplitude peak values of both $S(u)$ and $K(u)$ in the outer region consistently occur

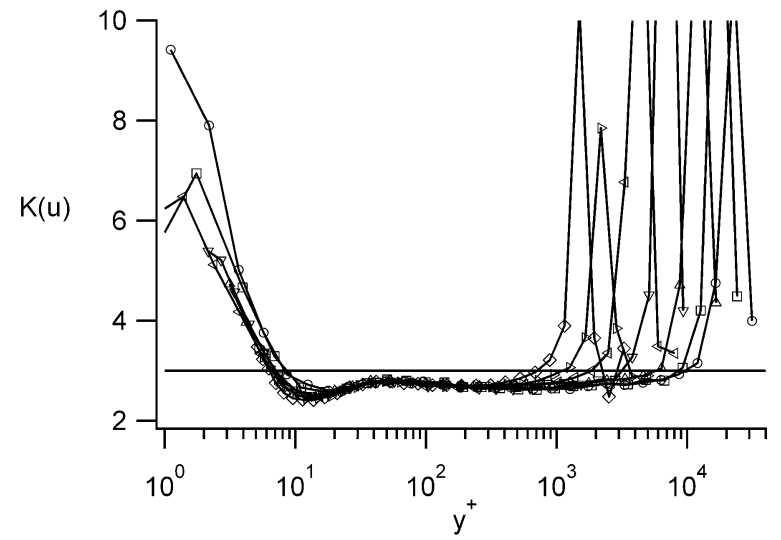

Fig. 18 Profiles of the streamwise velocity kurtosis plotted versus $y^{+}$for the primary data sets. Symbols are given in Table 2 .

almost identically at $\delta_{99}$, as estimated from the mean velocity profile.

\subsection{Streamwise Velocity Spectra}

The mid-layer peak in the two-dimensional spectrogram of the $u$ fluctuations is a key observation of the past decade (Marusic et al., 2010; Klewicki, 2010). This spectral peak has been shown to underlie the emergence of the broadband peak seen in Fig. 12, and constitutes the signature of the increasingly energetic superstructure motions that, on average, reside just beyond $y^{+} \simeq$ $2.6 \sqrt{\delta^{+}}$, e.g., Hutchins \& Marusic $(2007 \mathrm{a}, \mathrm{b})$; Mathis et al. (2009). Owing to either facility size or computational box size constraints, very few studies have been able to document the emergence of this spectral peak by increasing $x$ in a single developing boundary layer at fixed $U_{\infty}$

Figure 19 shows a sequence of two-dimensional spectrograms from the primary data sets. Each of these presents a contour plot of the premultipled $u$ spectra, $\Psi_{u}=k_{x} \phi_{u}$, versus $\lambda^{+}$on the vertical axis and $y^{+}$ on the horizontal axis. Here the streamwise wavenumber and wavelength, $k_{x}$ and $\lambda$, are estimated using the local mean velocity. The Reynolds number varies by over a factor of five from the first to last frame in Fig. 19, beginning with $\delta^{+}=3,820$ and ending with $\delta^{+}=19,670$. The first three frames are for increases in $\delta^{+}$solely by increasing the $x$ location. A unique feature of the present data sets is their capacity to fully capture the inner peak region. These data convincingly reveal that the $\left(y^{+}, \lambda^{+}\right)$extent of the region immediately surrounding the inner peak is essentially invariant over the Reynolds number range of present study. It is observed, however, that the large wavelength extent of the spectrogram at $y^{+} \simeq 15$ increases in scale approximately in 


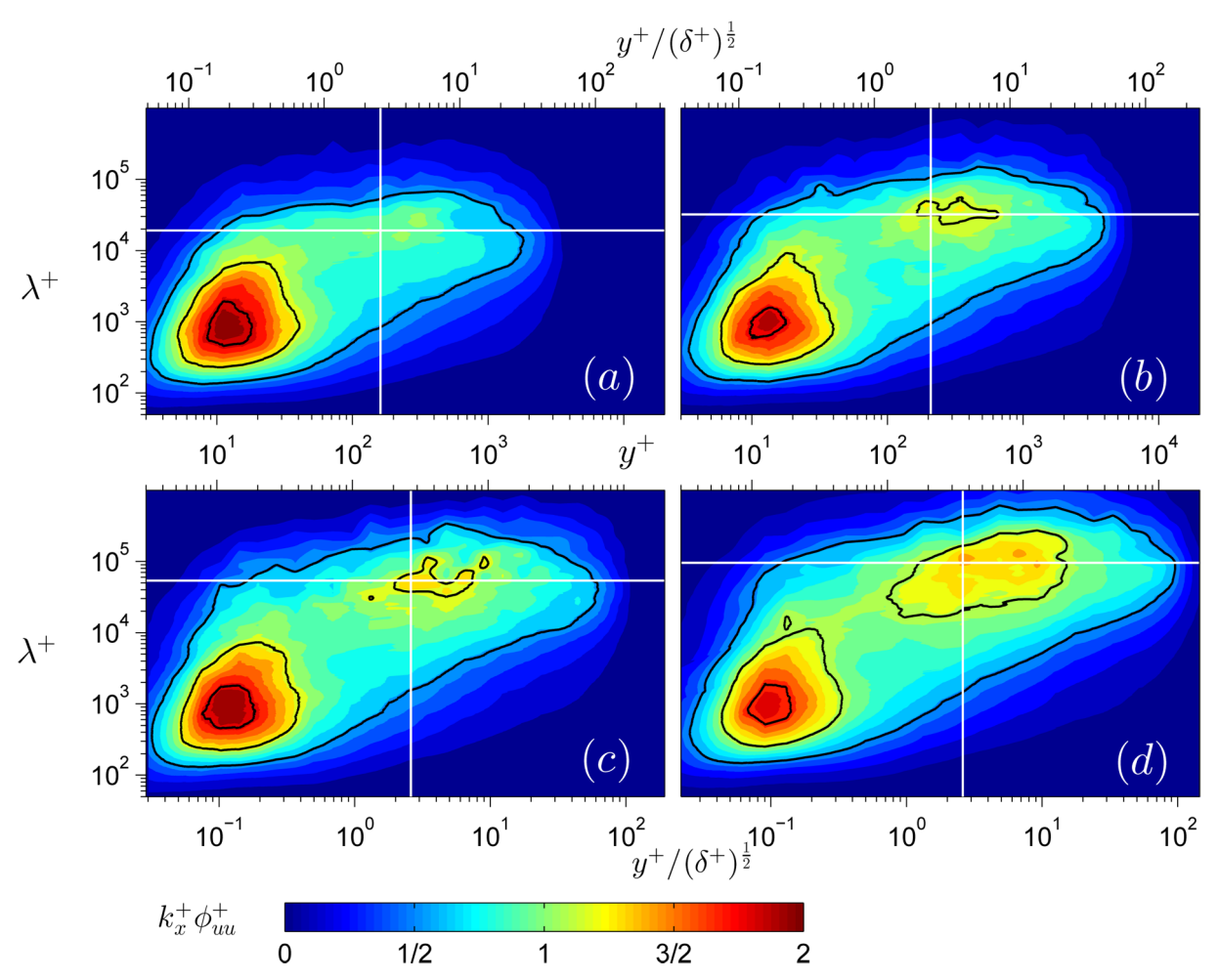

Fig. 19 Two dimensional spectrograms of the $u$ fluctuations, (a) $\delta^{+}=3,820$, (b) $\delta^{+}=6,430,(\mathrm{c}) \delta^{+}=10,770,(\mathrm{~d})$ $\delta^{+}=19,670$. Vertical line on each frame denotes $y^{+} / \sqrt{\delta^{+}}=2.6$. The horizontal line denotes $\lambda / \delta=5$.

proportion to the $\lambda^{+}$position of the mid-layer peak, i.e., at $y^{+}=15$ observe the position of the horizontal white line relative to outer solid line contour of the spectrogram. Similarly, the spectrogram appears to indicate that, for the $\delta^{+}$range explored, this wavelength retains energetic dominance out to the edge of the boundary layer. Relative to the development of the mid-layer spectral peak, the present results also seem to indicate that the most rapid change occurs for the $\delta^{+}$doubling between $\delta^{+}=10,770$ and $\delta^{+}=19,670$. Consistent with previous observations, the mid-layer spectral peak is nominally centered about $y^{+} \simeq 2.6 \sqrt{\delta^{+}}$(denoted by the vertical line on each frame), and $\lambda^{+} \simeq 5 \delta^{+}$, e.g., Hutchins \& Marusic (2007a).

\section{Conclusions}

Measurements of streamwise velocity statistics up to fourth central moment were acquired in turbulent boundary layers over the range, $1,450 \leq \delta^{+} \leq 19,670$. The measurements were acquired at low speed, and thus even the present $1 \mathrm{~mm}$ sensor had very good spatial and temporal resolution. Owing to the uniquely long development length afforded by the FPF test section, about half of the Reynolds number range was attained by increasing $x$, rather than increasing $U_{\infty}$. Comparison with the results from the HRNBLWT suggest that the development of $\delta^{*} / \delta$ and $\theta / \delta$ (Figs. 7 and 8) may contain an effect associated with the state of the boundary layer when it is tripped. On the other hand, the results of Fig. 9 indicate that the influence of this offset is essentially removed if $H=\delta^{*} / \theta$ is employed. Thus, except for an offset that seems to be associated with boundary layer growth upstream of the trip, the present findings are felt to provide strong support for self-similar $\delta^{*}$ and $\theta$ development.

The present results suggest that the onset of the logarithmic region is at a wall-normal location that, with increasing $\delta^{+}$, moves outward under inner normalization and inward under outer normalization (Fig. 6). This is in accord with other recent measurements at high Reynolds number (Marusic et al., 2013), and with theoretical predictions based upon the scaling properties admitted by the mean momentum equation (Fife et al., 2009; Klewicki, 2013a). In this regard, the intermediate scaling of the mean velocity gradient supports the theoretical finding that the onset of logarithmic behavior occurs at $y^{+} \simeq 2.6 \sqrt{\delta^{+}}$, which is physically where the mean viscous force in the mean dynamical equation loses leading order.

The near-wall peak in $\left\langle u^{2}\right\rangle^{+}$was shown to increase in accord with previous well-resolved measurements. The present measurements suggest the emergence of 
a mid-layer peak in $\left\langle u^{2}\right\rangle^{+}$, that corresponds with the more well-confirmed mid-layer spectral peak (see Figs. 14 and 19). Both of these peaks were shown to be located near $y^{+} \simeq 2.6 \sqrt{\delta^{+}}$, as was the mid-layer zero-crossing of $S(u)$ (Fig. 16). Extrapolations suggest that the midlayer peak of $\left\langle u^{2}\right\rangle^{+}$will become larger than the inner peak at $\delta^{+} \simeq 1 \times 10^{6}$, but existing field data indicate that the Reynolds number when this occurs, if it occurs, is larger. In accord with the results of Marusic et al. (2013), the present measurements support the emergence of a logarithmic decay in $\left\langle u^{2}\right\rangle^{+}$over the same domain as where the mean profile exhibits a logarithmic increase. The inner peak region of the two dimensional spectrogram of $u$ was shown to be invariant with Reynolds number in scale and position for the $\delta^{+}$range explored. The large wavelength tail of the spectrum at $y^{+} \simeq 15$, however, increases like $\lambda / \delta \simeq 5$, which correlates with the wavelength of the mid-layer spectral peak. The present spectrograms provide evidence that this wavelength also persists as the most energetic from the mid-layer peak to the edge of the boundary layer.

Acknowledgements This work was partially supported by the National Science Foundation, and by the Office of Naval Research. The authors are grateful to Dr. V. Kulandaivelu for making his data available.

\section{References}

Coles, D.E. (1956) The law of the wake in the turbulent boundary layer. J. Fluid Mech. 1: 191-226.

DeGraaff, D.B. and Eaton, J.K. (2000) Reynolds number scaling of the flat plate turbulent boundary layer. J. Fluid Mech. 422: 319-346.

Fife, P., Wei, T., Klewicki, J. and McMurtry, P. (2005) Stress gradient balance layers and scale hierarchies in wall-bounded turbulent flows. J. Fluid Mech. 532: 165-189.

Fife, P., Klewicki, J. and Wei, T. (2009) Time averaging in turbulence settings may reveal an infinite hierarchy of length scales. J. of Discrete and Continuous Dynamical Systems 24: 781-807.

Gad-el-Hak, M., \& Bandyopadhyay, P. (1994) Reynolds number effects in wall-bounded turbulent flows. Appl. Mech. Rev. 47: 307-365.

Hultmark, M., Vallikivi, M., Bailey, S.C.C. (2012) Turbulent pipe flow at extreme Reynolds numbers. Phys. Rev. Lett. 108: 094501.

Hutchins, N. \& Marusic, I. (2007a) Large-scale influences in near-wall turbulence. Phil. Trans. Roy. Soc. Lond. A 365: 647-664.

Hutchins, N. \& Marusic, I. (2007b) Evidence of very long meandering streamwise structures in the loga- rithmic region of turbulent boundary layers. J. Fluid Mech. 579: 1-28.

Hutchins, N., Nickels, T.B., Marusic, I. \& Chong, M.S. (2009) Hot-wire spatial resolution issues in wallbounded turbulence. J. Fluid Mech. 635: 103-136.

Hutchins N. (2012) Caution: tripping hazards. J. Fluid Mech. 710: 1-4.

Johansson, A.V. and Alfredsson, P.H. (1983) Effects of imperfect spatial resolution on measurements of wallbounded shear flows. J. Fluid Mech. 137: 409-421.

Klewicki, J. and Falco, R. (1990) On accurately measuring statistics associated with small-scale structure in turbulent boundary layers using hot-wire probes. J. Fluid Mech. 219: 119-142.

Klewicki, J., Fife, P., Wei, T. \& McMurtry, P. (2007) A physical model of the turbulent boundary layer consonant with mean momentum balance structure Phil. Trans. Roy. Soc. A 365: 823-839.

Klewicki, J. (2010) Reynolds number dependence, scaling and dynamics of turbulent boundary layers. $J$. Fluids Eng. 132: 094001.

Klewicki, J., Fife, P. and Wei, T. (2009) On the logarithmic mean profile. J. Fluid Mech. 638: 73-93.

Klewicki, J.C. (2013a) Self-similar mean dynamics in turbulent wall-flows. J. Fluid Mech. 718: 596-621.

Klewicki, J.C. (2013b) A description of turbulent wallflow vorticity consistent with mean dynamics. $J$. Fluid Mech. (submitted).

Kulandaivelu, V.(2012) Evolution of zero pressure gradient turbulent boundary layers from different initial conditions. PhD Dissertation, University of Melbourne.

Ligrani, P.M. and Bradshaw, P. (1987) Spatial Resolution and Measurement of Turbulence in the Viscous Sublayer Using Subminiature Hot-Wire Probes. Expts. Fluids 5: 407-417.

Marusic, I., McKeon, B.J., Monkewitz, P.A., Nagib, H.M., Smits, A.J. \& Sreenivasan, K.R. (2010) Wallbounded turbulent flows: recent advances and key issues. Phys. Fluids 22: 065103.

Marusic, I., Monty, J.P., Hultmark, M. and Smits, A.J. (2013) On the logarithmic region in wall turbulence. J. Fluid Mech. 716: R3.

Mathis, R., Hutchins, N., \& Marusic, I. (2009) Largescale amplitude modulation of the small-scale structures in turbulent boundary layers. J. Fluid Mech. 628: 311-337.

Mathis, R., Marusic, I., Hutchins, N., \& Sreenivasan, K.R. (2012) The relationship between the velocity skewness and the amplitude modulation of the small scale by the large scale in turbulent boundary layers. Phys. Fluids 23: 121702. 
Metzger, M.M. and Klewicki, J.C. (2001) A comparative study of near-wall turbulence in high and low Reynolds number boundary layers. Phys. Fluids. 13: 692-701.

Mochizuki, S. \& Nieuwstadt, F.T.M. (1996) Reynoldsnumber-dependence of the maximum in the streamwise velocity fluctuations in wall turbulence. Expts. Fluids. 21: 218-226.

Monkewitz, P.A., Chauhan, K.A., and Nagib, H.M. (2008) Approach to an asymptotic state for zero pressure gradient turbulent boundary layers. Phil. Trans. Roy Soc. A 365: 755-770.

Morrill-Winter, C. \& Klewicki, J. (2013) Scale separation effects on the mean vorticity transport mechanism of wall turbulence. Phys. Fluids 25: 015108.

Nagib, H.M., Chauhan, K.A., and Monkewitz, P.A. (2007) Comparisons of mean flow similarity laws in zero pressure gradient turbulent boundary layers. Phys. Fluids 20: 105102.

Patel, V.C. (1965) Calibration of the Preston tube and limitations on its use in pressure gradients. J. Fluid Mech. 23: 185-208.

Priyadarshana, P.J.A., Klewicki, J.C., Treat, S. \& Foss, J.F. (2007) Statistical structure of turbulentboundary layer velocity-vorticity products at high and low Reynolds numbers. J. Fluid Mech. 570: 307346.

Pullin, D., Inoue, M. \& Saito, N. (2013) On the asymptotic state of high Reynolds number, smoothwall turbulent flows. Phys. Fluids 25: 015116.

Schlatter, P. and Orlu, R. (2012) Turbulent boundary layers at moderate Reynolds numbers: inflow length and tripping effects. J. Fluid Mech. 710: 5-34.

Smits, A.J., McKeon, B.J. and Marusic, I. (2011) High Reynolds number wall turbulence. Ann. Rev. Fluid Mech. 43: 353-375.

Wei, T., Fife, P., Klewicki, J. \& McMurtry, P. (2005) Properties of the mean momentum balance in turbulent boundary layer, pipe and channel flows. J. Fluid Mech. 522: 303-327. 